\title{
AN EXTENSION OF NEUMANN'S INTEGRALRELATION FOR GENERALIZED LEGENDRE FUNCTIONS
}

By H. S. V. DE SNoo

In this paper we obtain an integralrelation connecting the two linearly independent generalized Legendre functions of Kuipers and Meulenbeld. The result is a generalization of F. Neumann's relation of 1848 for the two kinds of Legendre functions

$$
Q_{k}(z)=\frac{1}{2} \int_{-1}^{1} \frac{P_{k}(x)}{z-x} d x
$$

where $k$ is a nonnegative integer, and $z$ is not lying on the segment $(-1,1)$ of the complex plane.

The main result is in $\$ 2$; generalizations can be found in $\$ 4$. E. R. Love's integralrelations of 1965 for associated Legendre functions follow as special cases.

1. The generalized Legendre functions $P_{k}^{m, n}(z)$ and $Q_{k}^{m, n}(z)$, two specified linearly independent solutions of the differential equation

$$
\left(1-z^{2}\right) \frac{d^{2} w}{d z^{2}}-2 z \frac{d w}{d z}+\left\{k(k+1)-\frac{m^{2}}{2(1-z)}-\frac{n^{2}}{2(1+z)}\right\} w=0,
$$

have been introduced by Kuipers and Meulenbeld [3] as functions of $z$ for all points of the $z$-plane, in which a cross-cut exists along the real $x$-axis from 1 to $-\infty$, and for complex values of the parameters $k, m$ and $n$. On the segment $-1<x<1$ of the cross-cut these functions are defined in [7]. If $m=n$, they reduce to the associated Legendre functions, defined in [2].

For the sake of brevity we put

$$
\begin{array}{ll}
\alpha=k+\frac{1}{2}(m+n), & \beta=k-\frac{1}{2}(m-n), \\
\gamma=k+\frac{1}{2}(m-n), & \delta=k-\frac{1}{2}(m+n) .
\end{array}
$$

Generalized Legendre functions can be written in terms of hypergeometric functions, such as $[4,(9)]$

$$
\begin{aligned}
Q_{k}^{m, n}(z)=e^{\pi i m} 2^{\beta} \frac{\Gamma(\alpha+1) \Gamma(\gamma+1)}{\Gamma(2 k+2)}(z+1)^{-k+\frac{1}{3} m-1}(z-1)^{-\frac{1}{2} m} & \\
& \cdot F\left(\beta+1, \delta+1 ; 2 k+2 ; \frac{2}{1+z}\right)
\end{aligned}
$$

if $z$ is not lying on the cross-cut.

Received March 7, 1968. The author wishes to express his indebtedness to Professors R. L. van de Wetering and B. Meulenbeld. 
2. The extension of Neumann's integral is contained in the following theorem.

Theorem 1. If $\operatorname{Re} m<1, \operatorname{Re} \alpha>-1, \operatorname{Re} \gamma>-1$, and $z$ is not lying on the cross-cut, then

$$
\frac{(z+1)^{k+\frac{1}{2} m}}{(z-1)^{\frac{1}{2} m}} e^{-m \pi i} Q_{k}^{m, n}(z)=\frac{1}{2} \int_{-1}^{1} \frac{(1+x)^{k+\frac{1}{2} m}}{(1-x)^{\frac{1}{2} m}} \frac{P_{k}^{m, n}(x)}{z-x} d x .
$$

Proof. In $[8,(13)]$ one can find the integral transform

$$
\begin{aligned}
& \int_{-1}^{1}(1-x)^{-\frac{1}{2} m}(1+x)^{q}(z+x)^{-\sigma} P_{k}^{m, n}(x) d x \\
& =2^{q+\frac{1}{2} n-m+1} \frac{\Gamma\left(q+\frac{1}{2} n+1\right) \Gamma\left(q-\frac{1}{2} n+1\right)}{\Gamma\left(q-k-\frac{1}{2} m+1\right) \Gamma\left(q+k-\frac{1}{2} m+2\right)}(z-1)^{-\sigma} \\
& \quad \cdot{ }_{3} F_{2}\left(q+\frac{1}{2} n+1, \sigma, q-\frac{1}{2} n+1 ;\right. \\
& \left.\quad q-k-\frac{1}{2} m+1, q+k-\frac{1}{2} m+2 ; \frac{2}{1-z}\right)
\end{aligned}
$$

if $\operatorname{Re} m<1, \operatorname{Re} q+1>\frac{1}{2}|\operatorname{Re} n|, z$ not lying on the cross-cut.

Choose $\sigma=1, q=k+\frac{1}{2} m$ and replace $z$ by $-z$ in (3); then

$$
\begin{aligned}
& \int_{-1}^{1} \frac{(1+x)^{k+\frac{1}{2} m}}{(1-x)^{\frac{1}{2} m}} \frac{P_{k}^{m, n}(x)}{z-x} d x \\
& \quad=2^{\beta+1} \frac{\Gamma(\alpha+1) \Gamma(\gamma+1)}{\Gamma(2 k+2)}(z+1)^{-1} F\left(\alpha+1, \gamma+1 ; 2 k+2 ; \frac{2}{1+z}\right)
\end{aligned}
$$

if $\operatorname{Re} m<1, \operatorname{Re} \alpha>-1, \operatorname{Re} \gamma>-1, z$ not lying on the cross-cut.

Using (1) and the relation [5, (6)]

$$
Q_{k}^{-m,-n}(z)=e^{-2 m \pi i} 2^{m-n} \frac{\Gamma(\beta+1) \Gamma(\delta+1)}{\Gamma(\alpha+1) \Gamma(\gamma+1)} Q_{k}^{m, n}(z)
$$

we obtain

$$
\begin{aligned}
F(\alpha+1, \gamma & \left.+1 ; 2 k+2 ; \frac{2}{1+z}\right) \\
& =e^{-m \pi i} 2^{-\beta} \frac{\Gamma(2 k+2)}{\Gamma(\alpha+1) \Gamma(\gamma+1)}(z+1)^{k+\frac{1}{2} m+1}(z-1)^{-\frac{1}{2} m} Q_{k}^{m, n}(z) .
\end{aligned}
$$

By setting (6) in (4) we complete the proof of the theorem.

Remark 1. The above theorem is a generalization of results of Meulenbeld and Robin [9, (30) and (31)]. After some simplifications Neumann's integral follows from Theorem 1 for $m=n=0$ and nonnegative integer $k$.

3. In this section we derive two lemmas, in order to generalize Theorem $\mathbf{1 .}$ 
Lemma 1. If $\operatorname{Re} m<1$ and $j$ is an integer satisfying

$$
0 \leq j<\min (\operatorname{Re} \alpha, \operatorname{Re} \gamma)
$$

then

$$
\int_{-1}^{1} \frac{(1+x)^{k+\frac{1}{2} m-j-1}}{(1-x)^{\frac{1}{2} m}} P_{k}^{m, n}(x) d x=0 .
$$

Proof. In [1, (8.1)] we find the integraltransform

$$
\begin{aligned}
\int_{-1}^{1} & (1-x)^{p}(1+x)^{a} P_{k}^{m, n}(x) d x \\
= & \frac{\Gamma\left(q+\frac{1}{2} n+1\right) \Gamma\left(p-\frac{1}{2} m+1\right)}{\Gamma(1-m) \Gamma\left(p+q-\frac{1}{2}(m-n)+2\right)} 2^{p+a-\frac{1}{2}(m-n)+1} \\
& \cdot{ }_{3} F_{2}\left(\beta+1,-\gamma, p-\frac{1}{2} m+1 ; 1-m, p+q-\frac{1}{2}(m-n)+2 ; 1\right)
\end{aligned}
$$

if $\operatorname{Re}\left(p-\frac{1}{2} m\right)>-1, \operatorname{Re} q+1>\frac{1}{2}|\operatorname{Re} n|$. Substitute $p=-\frac{1}{2} m, q=k+$ $\frac{1}{2} m-j-1$ in (8); then

$$
\begin{aligned}
\int_{-1}^{1}(1-x)^{-\frac{1}{2} m}(1+x)^{k+\frac{1}{2} m-i-1} P_{k}^{m, n}(x) d x \\
=2^{\alpha-j} \frac{\Gamma(\alpha-j)}{\Gamma(\beta-j+1)} F(\beta+1,-\gamma ; \beta-j+1 ; 1)
\end{aligned}
$$

if $\operatorname{Re} m<1, \operatorname{Re} j<\min (\operatorname{Re} \alpha, \operatorname{Re} \gamma)$. Using [2, 2.8 (46)] the right-hand side of (9) reduces to

$$
2^{\alpha-i} \frac{\Gamma(\alpha-j) \Gamma(\gamma-j)}{\Gamma(-j) \Gamma(-j+2 k+1)}
$$

which is equal to 0 for $j$ satisfying the conditions of Lemma 1.

Lemma 2. If $\operatorname{Re} m<1, j$ is any integer satisfying

$$
0 \leq j<\min (\operatorname{Re} \alpha, \operatorname{Re} \gamma)
$$

and $p(x)$ is any polynomial of degree $j$ or less, then

$$
\int_{-1}^{1} \frac{(1+x)^{k+\frac{1}{2} m-i-1}}{(1-x)^{\frac{1}{2} m}} P_{k}^{m, n}(x) p(x) d x=0 .
$$

Proof. Let $p(x)$ be a polynomial of degree $j$ or less. Then $p(x)$ can be written in the form

$$
p(x)=\sum_{i=0}^{i} p_{i}(1+x)^{i-i}
$$

This yields

$$
\int_{-1}^{1} \frac{(1+x)^{k+\frac{1}{2} m-i-1}}{(1-x)^{\frac{1}{2} m}} p(x) P_{k}^{m, n}(x) d x=\sum_{i=0}^{j} p_{i} \int_{-1}^{1} \frac{(1+x)^{k+\frac{1}{2} m-i-1}}{(1-x)^{\frac{1}{3} m}} P_{k}^{m, n}(x) d x .
$$

By virtue of Lemma 1 the right-hand side vanishes. 
4. This section contains two generalizations of Theorem 1. The proofs presented are similar to those in [6].

Theorem 2. If $\operatorname{Re} m<1, \operatorname{Re} \alpha>-1, \operatorname{Re} \gamma>-1, z$ is not lying on the cross-cut, $s$ is an integer satisfying

$$
0 \leq s<\min (\operatorname{Re} \alpha+1, \operatorname{Re} \gamma+1)
$$

and $p(x)$ is a polynomial of degree $s$ or less, then

$$
\frac{(z+1)^{k+\frac{1}{2} m-8}}{(z-1)^{\frac{1}{2} m}} e^{-m \pi i} Q_{k}^{m, n}(z) p(z)=\frac{1}{2} \int_{-1}^{1} \frac{(1+x)^{k+\frac{1}{2} m-s}}{(1-x)^{\frac{1}{2} m}} \frac{P_{k}^{m, n}(x)}{z-x} p(x) d x .
$$

Proof. Using Theorem 1 and subtracting the right-hand side from the left-hand side in (11) we obtain

$$
\begin{aligned}
& \frac{p(z)}{(z+1)^{8}} \frac{1}{2} \int_{-1}^{1} \frac{(1+x)^{k+\frac{1}{2} m}}{(1-x)^{\frac{1}{2} m}} \frac{P_{k}^{m, n}(x)}{z-x} d x-\frac{1}{2} \int_{-1}^{1} \frac{(1+x)^{k+\frac{1}{2} m}}{(1-x)^{\frac{1}{2} m}} \frac{P_{k}^{m, n}(x)}{z-x} \frac{p(x)}{(1+x)^{s}} d x \\
& =\frac{1}{2} \int_{-1}^{1} \frac{(1+x)^{k+\frac{1}{2} m-s}}{(1-x)^{\frac{1}{2} m}} P_{k}^{m, n}(x)\left\{\frac{p(z)}{(z+1)^{s}} \frac{(1+x)^{s}-(z+1)^{s}}{z-x}+\frac{p(z)-p(x)}{z-x}\right\} d x \\
& =0
\end{aligned}
$$

because for $1 \leq s<\min (\operatorname{Re} \alpha+1, \operatorname{Re} \gamma+1)$ the expression between braces is a polynomial in $x$ of degree $s-1$, so that we can apply Lemma 2 by putting $j=s-1$. For $s=0$ the polynomial $p(x)$ reduces to a constant, and (11) follows from Theorem 1 .

Remark 2. The above theorem is a generalization of results of Meulenbeld and Robin [9, (53) and (55)].

Theorem 3. If $\operatorname{Re} m>-1, \operatorname{Re} \beta>-1, \operatorname{Re} \delta>-1, \alpha$ is not an integer, $z$ is not lying on the cross-cut, $s$ is an integer satisfying

$$
0 \leq s<\min (\operatorname{Re} \beta+1, \operatorname{Re} \delta+1)
$$

and $p(x)$ is any polynomial of degree $s$ or less, then

$$
\begin{aligned}
\frac{(z+1)^{k-\frac{1}{2} m-s}}{(z-1)^{-\frac{1}{2} m}} e^{-m \pi i} Q_{k}^{m, n}(z) p(z) & \\
= & \frac{1}{2 \sin \alpha \pi}\left\{\sin \beta \pi \int_{-1}^{1} \frac{(1+x)^{k-\frac{1}{3} m-s}}{(1-x)^{-\frac{1}{2} m}} \frac{P_{k}^{m, n}(x)}{z-x} p(x) d x\right. \\
& \left.+2^{n-m} \sin m \pi \frac{\Gamma(\gamma+1)}{\Gamma(\beta+1)} \int_{-1}^{1} \frac{(1-x)^{k-\frac{1}{2} m-s}}{(1+x)^{-\frac{1}{2} m}} \frac{P_{k}^{n, m}(x)}{z+x} p(-x) d x\right\}
\end{aligned}
$$

Proof. By combining [7, (8)] and [7, (10)] we obtain, if $\alpha$ is not integer

$$
\begin{aligned}
& \frac{\Gamma(\alpha+1) \Gamma(\gamma+1)}{\Gamma(\beta+1) \Gamma(\delta+1)} P_{k}^{-m,-n}(x) \\
& =\frac{1}{\sin \alpha \pi}\left(2^{m-n} \sin \beta \pi P_{k}^{m, n}(x)+\sin m \pi \frac{\Gamma(\gamma+1)}{\Gamma(\beta+1)} P_{k}^{n, m}(-x)\right) .
\end{aligned}
$$


In Theorem 2 replace $m$ by $-m$ and $n$ by $-n$, use (5) for the left-hand side and (13) for the right-hand side, split up the integral in the right-hand side (this is allowed because both integrals exist under given conditions) and in the integral involving $P_{k}^{n, m}(-x)$ change $x$ into $-x$. This completes the proof.

Remark 3. All results given in this paper reduce to E. R. Love's results [6] by setting $m=n$. For references to special cases in the case of associated Legendre functions, we refer to [6].

\section{References}

1. B. L. J. BraAksma AND B. Meulenbeld, Integral transforms with generalized Legendre functions as kernels, Compositio Math., vol. 18(1967), pp. 235-287.

2. A. Erdelyi, Higher Transcendental Functions I, New York-Toronto-London, 1953.

3. L. Kuipers AND B. Meulenbeld, On a generalization of Legendre's associated differential equation, I and II, Proc. Kon. Ned. Akad. Wet., Amsterdam, vol. 60(1957), pp. 436-450.

4. L. Kuipers AND B. Meulenbeld, Linear transformations of generalized Legendre's associated functions, Proc. Kon. Ned. Akad. Wet., Amsterdam, vol. 61(1958), pp. $330-333$.

5. L. Kuipers and B. Meulenbeld, Some properties of a class of generalized Legendre's associated functions, Proc. Kon. Ned. Akad. Wet., Amsterdam, vol. 61(1958), pp. 186-197.

6. E. R. Love, Franz Neumann's integral of 1848, Proc. Cambridge Philos. Soc., vol. 61(1965), pp. $445-456$.

7. B. Meulenbeld, Generalized Legendre's associated functions for real values of the argument numerically less than unity, Proc. Kon. Ned. Akad. Wet., Amsterdam, vol. 61(1958), pp. $557-563$.

8. B. Meulenbeld ANd H. S. V. DE SNoo, Integrals involving generalized Legendre functions, J. Engrg. Math., vol. 1(1967), pp. 285-291.

9. B. Meulenbeld and L. Robin, Nouveaux résultats relatifs aux fonctions de Legendre généralisées, Proc. Kon. Ned. Akad. Wet., Amsterdam, vol. 64(1961), pp. 333-347.

Delft Institute of Technology

San Diego State College 